\title{
Beitrag zur Optimierung der Datengrundlagen für die Sanierungsplanung anhand einer Prozessanalyse bei der baulichen Zustandserfassung durch Kanal-TV-Inspektionen
}

\begin{abstract}
Zusammenfassung: Der kurz- bis mittelfristige Sanierungsbedarf von Kanalisationen wird in Bayern auf 4 bis 5 Mrd. Euro geschätzt. In Österreich kann man aufgrund von vergleichbaren siedlungswasserwirtschaftlichen Strukturen von ähnlichen Dimensionen ausgehen. Die

Die Erfüllung der Pflichten des/der Auftraggebers/Auftraggeberin wie z. B. die Überprüfung der Erbringung der vertraglichen Leistungen können durch die vorgeschlagenen begleitenden qualitätssicherndenMaßnahmensehrkosteneffizient erbracht werden.
\end{abstract} TV-Inspektion als Mittel der Zustandserfassung bei Kanalisationsanlagen liefert wesentliche Grundlagen für die Sanierungsplanung von Entwässerungssystemen. Trotz der daraus resultierenden enormen volkswirtschaftlichen Bedeutung liegen die Marktpreise für TV-Inspektionen heute oftmals tiefer als die Kosten, die man für eine qualitativ hochwertige, vollständige und richtige Zustandserfassung berechnen kann. Das Sparen bei der Grundlagenermittlung der Kanalsanierung führt zwangsläufig zu Daten minderer Qualität, die als Basis für eine effiziente Sanierungsplanung vielfach unzureichend sind. Dieser Beitrag soll zeigen, dass die bauliche Zustandsbewertung basierend auf einer qualitätsgesicherten TV-Inspektion im Vergleich zu herkömmlichen Vorgehensweisen bei der Zustandsbewertung sogar durchaus finanzielle Vorteile für den Auftraggeber bieten könnte. Es werden dazu die Arbeitsschritte im Zuge der baulichen Zustandsbewertung und deren Kosten analysiert und verschiedene Vorgehensweisen zur Qualitätssicherung diskutiert. Die Ergebnisse zeigen, dass die Variante mit dem begleitenden Qualitätsmanagement gegenüber den relevanten Alternativen Vorteile mit sich bringt. Dabei können durch die begleitenden Überprüfungen sowie die regelmäßigen Schulungen des relevanten Personals die erforderlichen Qualitätsstandards bei der TV-Inspektion gewährleistet werden. Die berechneten Kosten sind theoretische Preise. Ausschreibungen der hier berechneten Varianten können aus verschiedenen Gründen davon abweichende Preise erzielen. Der Unterschied zu den Marktpreisen (bei Billigstbieterverfahren) ist in Relation zu den zu erwartenden Sanierungskosten aber vernachlässigbar, bei gleichzeitiger Steigerung der Qualität der erbrachten Leistungen.
Optimising basic data for rehabilitation planning using process analysis for sewer condition monitoring by TV inspections

Summary: The cost of short and medium term sewer rehabilitation requirements in Bavaria has been put at 4 to 5 billion euros. The fact that sewerage systems in Austria are comparable in structure suggests that the costs and requirements for these measures will be on a similar scale. As a means of studying the state of repair of sewers, TV inspection provides important data as a basis for planning the rehabilitation of sewerage systems. Despite the substantial economic importance involved, the market prices of TV inspections tend to be lower than the expense to be budgeted for complete and correct high-quality sewer condition monitoring. Economising in the determination of basic data for sewer rehabilitation necessarily results in data of lesser quality, which are often insufficient as a basis for efficient rehabilitation planning. This article is intended to demonstrate that the assessment of the state of repair of sewers based on quality-assured TV inspection may well offer financial advantages for the client as compared with conventional methods of ascertaining sewer condition. The authors analyse the the structural condition of sewers and the costs involved, and discuss different quality assurance approaches. The study shows that the variant providing for an accompanying quality management affords advantages over the relevant alternatives. The required quality standards of TV inspection could be safeguarded by the accompanying inspections and routine staff training. The calculated costs are theoretical prices. Bidding procedures for the variants calcuvarious steps in the process of assessing lated in this study may give different prices, though. The difference to the market prices (in the case of lowest-bidder tendering), however, is negligible in relation to the expected rehabilitation cost, while enhancing the quality of the work.

Furthermore a cost-efficient method of meeting the client's duties, such as inspection of the work performed under the contract, could be provided in the suggested accompanying quality-assurance measures.

\section{Einleitung}

„In Bayern sind rund 16\% der öffentlichen Kanalisation sanierungsbedürftig. Rund $12.500 \mathrm{~km}$ der Gesamtnetzlänge müssen kurz- bis mittelfristig saniert werden. Daraus resultiert ein Gesamtvolumen von rund 3,6 Mrd. Euro. Rechnet man die Sanierungskosten für Schächte und dem öffentlichen Teil der Hausanschlussleitungen hinzu, liegt das Volumen bei etwa 4 bis 5 Mrd. EUR“ (IKT, 2009). Da Österreich durchaus mit Bayern vergleichbare siedlungswasserwirtschaftliche Strukturen aufweist, kann man auch hierzulande von ähnlichen finanziellen Dimensionen bei der kurz- und mittelfristigen Kanalsanierung ausgehen. Die unterirdischen Kanalisationsanlagen stellen daher eines der größten Vermögenswerte der Kommunen dar, deren Erhalt bzw. Sanierung kommt eine große volkswirtschaftliche Bedeutung zu. Die TV-Inspektion dient primär der Erfassung des baulichen Zustandes von Entwässerungssystemen und liefert damit auch wesentliche Grundlagen für die Sanierungsplanung.

Es ist heute eine Vielzahl von qualitativ hochwertigen Inspektionstechnologien verfügbar, entsprechende Codierungscodes (ON EN 13508-2) zur Zustandsbeschreibung sind vorhanden, es werden Ausbildungskurse (z. B. ÖWAV) für das Inspektionspersonal angeboten. Nationale und internationale Erfahrungen (Kanfunk 2006; Korving und Clemens 2004, zit. bei Gangl et al. 2006; Plihal 2009) zeigen allerdings, dass die Qualität der TV-Inspektion 
oftmals noch verbessungswürdig ist. Bölke und Ertl (2007) nennen als mögliche Fehlerquellen u. a. den Faktor Mensch in Gestalt des Inspekteurs, der die Zustände vor Ort unter oft erschwerten Arbeitsbedingungen erkennen und dokumentieren muss.

Trotz der großen wirtschaftlichen Bedeutung liegen die Marktpreise für TV-Inspektionen heute oftmals tiefer als die Kosten, die man eigentlich bei einer qualitativ hochwertigen, vollständigen und richtigen Zustandserfassung als ausreichende Grundlage für die Sanierungsplanung erwarten würde. Auf die möglichen Gründe, die zu den teilweise als zu niedrig erscheinenden Marktpreisen führen, soll in diesem Beitrag aber nicht weiter eingegangen werden.

Die Laufmeterkosten einer Sanierung betragen oftmals mehr als das hundertfache der Laufmeterkosten einer TV-Inspektion (inkl. vorheriger Reinigung, $\mathrm{Zu}$ standsklassifizierung, etc.). Jung (2005) erklärt in diesem Zusammenhang, dass im Rahmen der Planung und Inspektion auf $65 \%$ der Kosten einer (unterirdischen) Sanierungsmaßnahme Einfluss genommen werden kann. Einsparungsmaßnahmen bei der TV-Inspektion auf Kosten der Datenqualität erscheinen dadurch mehr als kontraproduktiv.

Dieser Beitrag soll zeigen, dass das Sparen bei der TV-Inspektion als wesentliches Mittel der Grundlagenermittlung für die Kanalsanierung nicht im Sinne eines effizienten Kanalmanagements sein kann. Vielmehr könnte die baulicheZustandsbewertung basierend auf einer qualitätsgesicherten TVInspektion im Vergleich zu herkömmlichen Vorgehensweisen bei der Zustandsbewertung sogar durchaus finanzielle Vorteile für den Auftraggeber bieten.

Um den Zusammenhang zwischen Kosten und Qualitätssicherung entsprechend darstellen zu können, werden die Arbeitsschritte im Zuge der baulichen $\mathrm{Zu}$ standsbewertung von der Auftragsvergabe der Kanalinspektion bis hin zur Planung der Kanalsanierung und deren Kosten analysiert und verschiedene Vorgehensweisen zur Qualitätssicherung diskutiert. Es wird dabei die derzeit übliche Vorgehensweise mit allen ihren notwendigen Arbeitsschritten erläutert. Als alternative Varianten werden Szenarios vorgestellt, wie diese Arbeitsschritte die Qualität der Resultate verbessern und absichern könnten. Anhand einer Kosten-Nutzen-Analyse eines Beispielprojekts werden die verschieden Varianten verglichen, um zu zeigen, welche Auswirkungen und Opti- mierungsmöglichkeiten die einzelnen Variablen in Hinblick auf die Kosten einer Qualitätssicherung haben.

\section{Methodik}

\subsection{Grundannahmen}

Für die Kostenanalyse bei den unterschiedlichen Varianten der Qualitätssicherung wurden folgende Grundannahmen festgelegt:

a. Das Ergebnis der TV-Inspektion muss die Förderbestimmungen des Lebensministeriums (BMLFUW, 2006) für die Erstellung eines Leitungskataster erfüllen. Das bedeutet, dass neben der $\mathrm{Zu}$ standserfassung auch eine Zustandsbewertung durchgeführt werden muss. Im konkreten Fall wurde angenommen, dass die Zustandsbewertung bzw. Klassifizierung durch einen Ziviltechniker erfolgt, die dabei anfallenden Kosten wurden mit $1 € / \mathrm{lfm}$ angenommen.

b. Eine ausreichende Qualifikation des Personals für die TV-Inspektion sowie für die Überprüfung der fachlichen Richtigkeit der Inspektionsergebnisse wird bei allen Varianten vorausgesetzt.

c. Die inspizierten Kanäle werden als „sanierungsbedürftig“ angenommen. Es wird von einer durchschnittlichen Tagesleistung bei der TV-Inspektion von $600 \mathrm{~m}$ ausgegangen. Pro Haltung ist mit mindestens 10 Zuständen zu rechnen. Die Grundkosten für die TV-Inspektion (Fahrzeug mit 2 Personen) werden mit $100 €$ pro Stunde festgelegt. Bei 8 Arbeitsstunden pro Tag und $600 \mathrm{~m}$ zu inspizierenden Haltungen ergeben sich Laufmeterkosten für die TV-Inspektion von $1,33 €$. Die vor der Inspektion durchzuführende Hochdruckreinigung wird mit $1,5 € / \mathrm{lfm}$ festgelegt.

d. Die berechneten Kosten sind theoretische Preise. Ausschreibungen der hier berechneten Varianten können aus verschiedenen Gründen (wie z.B. Marktsituation, besondere Erschwernisse) davon abweichende Preise erzielen.

\subsection{Analyse der Arbeitsschritte}

Nach Koch (2009) erfolgt die Festlegung der Anforderungen an die Zustandserfassung durch den Auftraggeber im Rahmen der Ausschreibung, wobei die Qualitätssicherung von Inspektionsleistungen eine wesentliche Rolle spielt, aber derzeit nicht immer berücksichtigt wird. Daher sollten die Qualitätsvorgaben hinsichtlich technischem Equipment, Durchführung und Personal in zusätzlichen Vertragsbedingungen zusammengefasst werden. Die Intervalle für die Kanal-TV-Inspektion, die in Österreich je nach Bundesland zwischen 10 und 15 Jahren liegen, haben nach Winkler (2003) folgende Zielsetzung, wobei sich diese auf den Altbestand der Kanäle bezieht:

- Sanierungsnotwendigkeiten zu erkennen,

- Sanierungsstrategien zu entwickeln und

- konkrete Sanierungsmaßnahmen zu planen.

Für eine korrekte Sanierungsplanung ist eine zuverlässige Zustandserfassung durch die Kanalinspektion erforderlich.

\subsubsection{Beteiligte Akteurlnnen}

Von der Auftragsvergabe bis zur Sanierungsplanung sind unterschiedliche AkteurInnen beteiligt, die nachfolgend beschrieben werden.

AuftraggeberIn (AG): Dieser ist in den meisten Fällen die öffentliche Hand, z. B. VertreterIn der Gemeinde, des Verbandes oder des Magistrats. Die Auftragsvergabe kann von einer Firma kommen, die ihren Privatkanal inspizieren möchte, oder von teilprivatisierten Verbänden und Kommunen.

KonsulentIn (KO): Der KO ist der/die technische BeraterIn und PlanerIn des Auftraggebers und unterstützt diesen u. a. bei den folgenden Punkten:

- Entscheidungs- und Lösungsfindung von kanalrelevanten Themen

- Bereitstellung von Kanalplänen

- Kostenschätzung für Kanalerweiterungen

- Rechnungsprüfung

- Durchführung von Ausschreibung

- Einholung und Überprüfung von Angeboten

- Baustellenaufsicht

- Bindeglied zum Amt der Landesregierung

Kanalreinigungsfirma: Vor der Durchführung der Kanalinspektion für eine bauliche Zustandserfassung muss der Kanal gereinigt werden, wobei die Reinigung in Absprache mit der Inspektionsfirma erfolgen sollte. Einerseits darf der zeitliche Abstand zwischen Reinigung und Inspektion nicht zu groß sein, da der Kanal in der Zwischen- 
zeit wieder verschmutzen könnte. Andererseits darf der zeitliche Abstand auch nicht zu gering sein, da die Qualität der Inspektion durch die von nassen Rohrwänden erzeugten Spiegelungen oder Blendungen beeinträchtigt werden könnte. Die Vorgabe, wie groß der zeitliche Abstand (im Normalfall 12-24 Stunden) zwischen Reinigung und Inspektion idealerweise sein muss, sollte durch den/die Auftraggeber/Auftraggeberin bzw. KonsulentIn erfolgen.

Kanalinspektionsfirma (KI): Die Kanalinspektionsfirma ist der eigentliche Auftragnehmer, teilweise besitzen diese Firmen auch einen eigenen Reinigungsfuhrpark. Falls die Kanalinspektionsfirma auch die Kanalreinigung übernimmt, dann gilt es selbstverständlich auch hier, die zeitlichen Abstände, wie zuvor beschrieben, zu beachten.

Bei der Kanalinspektion sollten prinzipiell 2 entsprechend geschulte Personen vor Ort sein, wobei die zweite Person dann zwingend erforderlich ist, wenn ein Abstieg in den Kanal durchgeführt werden muss, wie z.B. bei Schachtinspektionsaufnahmen oder bei der Positionierung der Kamera in der Kanalsohle (vor allem wenn die Schächte als Fangschächte ausgebildet sind). Der Abstieg darf nur unter Einhaltung aller relevanten Vorschriften zur Arbeitssicherheit erfolgen (Absturzsicherung, Bergeeinrichtung, Befahrerlaubnisschein, verkehrstechnische Absicherung, etc.).

Qualitätsmanagement (QM): Das Qualitätsmanagement, das hinkünftig bei der Kanalinspektion integriert werden kann, hat die Aufgabe der Überwachung der Kanalinspektionsfirma in Bezug auf die Ausbildung des Inspektionspersonals, der Ausrüstung und des handwerklichen Umgangs mit den verwendeten Geräten. Dadurch sollen Fehler, die bei der Inspektion auftreten können, schon im Vorfeld minimiert werden und somit in weiterer Folge auch die Qualität der Sanierungsplanung verbessert werden. Die Funktionen des QM müssen durch gerichtlich beeidete Sachverständige oder gleichwertig qualifizierte Fachkräfte ausgeübt werden. Das Qualitätsmanagement kann dem Auftraggeber sowohl in beratender als auch in überprüfender Funktion zur Seite stehen.

\subsubsection{Arbeitsschritte}

Mit einer Kanalinspektion können unterschiedliche Ziele verfolgt werden (periodische oder bedarfsorientierte Inspektion,
Vorarbeiten zur Kanalsanierung, etc.). Der AG bespricht mit dem KO die Rahmenbedingungen für die notwendige Kanalinspektion und beauftragt anschließend den $\mathrm{KO}$, Angebote von mindestens 3 (besser 5) Kanalreinigungs- sowie Kanalinspektionsfirmen einzuholen. In weiterer Folge lässt der ZT von diesen Firmen Angebote erstellen, wobei er bzw. der AG zu Beginn Rahmenbedingungen für die Inspektion sowie die vorbereitenden Reinigung vorgibt, welche u. a. folgende Punkte enthalten können:

1. Definition der Art und des Zeitpunktes der vorbereitenden Reinigung:

2. Die Forderung an die Kanalinspektionsfirma, dass eine zweite, entsprechend geschulte Person bei der Inspektion vor Ort anwesend sein muss.

3. Definition eines für die Inspektion maximal zulässigen Wasserspiegels in den Haltungen sowie bei Überschreitung Hinweise auf das Umpumpen des anfallenden Abwassers.

4. Hinweise auf die richtige Handhabung der Inspektionsausrüstung (z. B. Objektiv mittig in der Rohrachse).

5. Der Hinweis auf die gesetzlichen Vorschriften, die bei einer erforderlichen „Befahrung“ des Kanals eingehalten werden müssen.

\subsection{Kosten-Nutzen-Analyse zur QM- Begleitung - Variantenvergleich}

Um die finanziellen Unterschiede zwischen den Varianten der herkömmlichen Vorgehensweisen bei der Zustandserfassung und -bewertung untereinander sowie mit begleitender Qualitätssicherung (QS) vergleichen zu können, wird eine Kosten-Nutzen-Analyse durchgeführt. Dabei werden die Kosten beginnend bei der Ausschreibung für die Kanalinspektion bis hin zur abschließenden Endklassifizierung als erste Grundlage für die Sanierungsplanung für die unterschiedlichen Varianten dar- bzw. gegenübergestellt.

In den nächsten beiden Kapiteln werden die wesentlichen Annahmen zur Datenquantifizierung sowie die untersuchten Varianten erörtert.

\subsubsection{Datengrundlagen und Annahmen}

Bei den untersuchten Varianten wird analysiert, wie sich die einzelnen Arbeitsbereiche der TV-Inspektion auf die Gesamtkosten auswirken und welche Konsequenzen dies nach sich zieht. Die heutzutage übliche Tagesleistung der Ka-

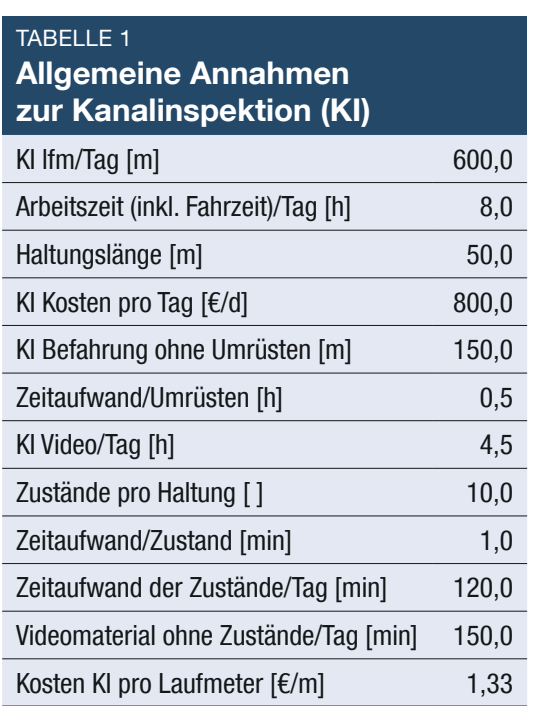

nalinspektion wird von den Kanalinspektionsfirmen mit $600 \mathrm{~m}$ bis $800 \mathrm{~m}$ angegeben. Dabei ist jedoch zu berücksichtigen, dass derartige Laufmeterleistungen erfahrungsgemäß nur dann erreicht werden können, wenn in den aufzunehmenden Strecken im Kanal auch größere Bereiche sind, die keine Schäden aufweisen. Bei den hier beschriebenen Untersuchungen wurde eine Tagesleistung von $600 \mathrm{~m}$ angenommen.

In Tabelle 1 sind die wesentlichen Annahmen zur Kostenberechnung der Kanal-TV-Inspektion bzw. Zustandserfassung (KI) ersichtlich.

Es wurde zusätzlich eine maximale Fahrgeschwindigkeit der Kamera von $4 \mathrm{~m} / \mathrm{min}$ angenommen. Die maximale Fahrgeschwindigkeit lt. Regelwerk DWA M149-2 (2007) beträgt zwar $15 \mathrm{~cm} / \mathrm{min}$ bzw. $9 \mathrm{~m} / \mathrm{min}$, im konkreten Fall wurde aber wegen der Verhältnisse in einem sanierungsbedürftigem Altbestand eine geringere Fahrgeschwindigkeit gewählt. Eine Inspektionsleistung von $600 \mathrm{~m}$ ergibt dann Videomaterial von 150 min für das Fahren ohne Dokumentation der Zustände. Bei angenommenen 10 erfassten Zuständen pro Haltung und 12 befahrenen Haltungen pro Tag ergibt dies Videomaterial der protokollierten Zustände von $120 \mathrm{~min}$ (bei $1 \mathrm{~min} /$ Zustand). Daraus ergeben sich in Summe $270 \mathrm{~min}(4,5 \mathrm{~h})$ Videomaterial pro Tag. Die restliche Tagesarbeitszeit wird für das Ein- und Ausbauen bzw. Umrüsten des Inspektionsgerätes (jeweils $0,5 \mathrm{~h}$ ) sowie für Fahrzeiten mit dem Auto veranschlagt. Bei der erwähnten Tagesinspektionsleistung sowie pauschal angenommenen Kosten von 800 ,- $€$ pro Tag ( $8 \mathrm{~h}$ mit $100 € / \mathrm{h}$ für Ausrüstung und 2 Personen) ergeben sich 


\begin{tabular}{lr}
\hline TABELLE 2 \\
Allgemeine Annahmen zur \\
Videokontrolle durch einen \\
(externen) Techniker \\
\hline Stundensatz [ $€ / d]$ \\
\hline Zeitfaktor für Begutachtung Video & 60,0 \\
\hline Anz. der h/Woche für Besprechungen & 2,0 \\
\hline Nachbesprechung mit AG [h] & 1,0 \\
\hline $\begin{array}{l}\text { Videokontrolle teilweise } \\
\text { (nur Zustände) [€/m] }\end{array}$ \\
\hline $\begin{array}{l}\text { Videokontrolle } \\
\text { restliches Videomaterial [ } € / \mathrm{m}]\end{array}$ \\
\hline
\end{tabular}

Laufmeterkosten für die Kanalinspektion von $1,33 €$.

Die wesentlichen Annahmen, die in Bezug auf den Arbeitsaufwand der Überprüfung der Ergebnisse der TV-Inspektion (Videokontrolle) durch einen (externen) Techniker getroffen wurden, sind in Tabelle 2 dargestellt.

Bei der Berechnung der Kosten pro Laufmeter wird davon ausgegangen, dass für die Begutachtung der Zustände die doppelte Zeit der Videolaufzeit benötigt wird (Zeitfaktor für die Begutachtung). $120 \mathrm{~min}$ Videomaterial mit $\mathrm{Zu}$ ständen ergeben daher einen Zeitaufwand von $240 \mathrm{~min}$ für den/die begutachtende/n TechnikerIn. Bei einem Stundensatz von $60 €$ und $600 \mathrm{~m}$ Tagesleitung der Kanalinspektion ergeben sich daraus Laufmeterkosten von $0,4 €$. Wird auch noch das Videomaterial der Befahrung ohne protokollierte Zustände (150 min) berücksichtigt (um die Erkennungsrate $\mathrm{zu}$ kontrollieren), ergeben sich Laufmeterkosten von 0,9€. Zu diesen Kosten sind noch die anteiligen Kosten für diverse Besprechungen zu addieren. Werden über den Zeitraum von einem Monat (20 Arbeitstage) pro Woche drei Stunden für Zwischenbesprechungen sowie eine Stunde für eine Endbesprechung berücksichtigt, so ergeben sich daraus zusätzliche Laufmeterkosten von rund $0.07 €$, die zu den oben genannten Kosten der Zustandsbegutachtung addiert werden.

In Tabelle 3 sind die wichtigsten Annahmen, die zur Berechnung des Aufwandes in Hinsicht auf ein begleitendes Qualitätsmanagement $(\mathrm{QM})$ getroffen wurden, dargestellt.

Beim begleitenden QM wird bei der Berechnung der Laufmeterkosten angenommen, dass die stichprobenartige Begutachtung der Inspektionsvideos die dreifache Zeit der eigentlichen Videolauf-

\begin{tabular}{lr}
\hline TABELLE 3 \\
Allgemeine Annahmen \\
zum begleitenden \\
Qualitätsmanagement (QM) \\
\hline Stundensatz QM [€/h] & 100,0 \\
\hline Zeitfaktor für Begutachtung Video & 3,0 \\
\hline $\begin{array}{l}\text { Begutachtung Videomaterial } \\
\text { stichprobe/Projekt [h] }\end{array}$ & 13,5 \\
\hline $\begin{array}{l}\text { Zeitaufwand Nachbesprechung } \\
\text { mit AG [h] }\end{array}$ & 4,0 \\
\hline Spesenersatz [\%] & 10,0 \\
\hline $\begin{array}{l}\text { Anteilige Kosten für unangekündigte } \\
\text { Baustellenbesuche [€] }\end{array}$ & 200,0 \\
\hline Anteilige Kosten für Schulungen [€] & 150,0 \\
\hline Kosten QM pro Ifm Kanal [€/m] & 0,19 \\
\hline
\end{tabular}

zeit beansprucht. Pro Inspektionsauftrag (angenommener Arbeitsumfang von 20 Tagen) werden jeweils nur die Inspektionsergebnisse eines beliebigen Tages überprüft. Durch das QM kann davon ausgegangen werden, dass die Qualität der Inspektionsergebnisse den Anforderungen an die Sanierungsplanung genügen. Dieses Qualitätsniveau soll neben der stichprobenartigen Überprüfung der Inspektionsvideos und -protokolle zusätzlich durch unangekündigt Baustellenbesuche sowie jährlicher Schulungen der Inspekteure erreicht werden.

Unter der Annahme eines Inspektionsauftrages mit der Dauer von 20 Arbeitstagen bei einem Stundensatz von $100 €$ für das QM, den Kosten für vier Besprechungsstunden je Auftrag, einem zusätzlichen Spesenersatz in der Höhe von 10\%, den anteiligen Kosten je Auftrag für unangekündigte Baustellenbesuche sowie den anteiligen Kosten je Auftrag für Schulungen ergeben sich für das begleitende QM Laufmeterkosten in der Höhe von rund $0,19 €$.

Neben den bisher beschrieben Punkten wurden für die Untersuchungen noch folgende Annahmen getroffen:

- Firma hat 200 Kanalinspektionstage pro Jahr bei 10 Aufträgen zu je 20 Tagen

- Kosten für die Ausschreibung und Vergabe in der Höhe von 1,0€/lfm

- Kanalreinigungsleistung $600 \mathrm{~m}$ pro Tag

- Kosten für die Kanalreinigung $800 €$ pro Tag (die Kosten für die Entsorgung des Räumguts wurden hier nicht berücksichtigt)

- Kosten für die Zustandsklassifizierung $1,0 € / \mathrm{lfm}$

\subsubsection{Untersuchte Varianten}

Folgende Varianten der Vorgehensweise bis zur Zustandsbewertung wurden definiert:

Variante 0: Diese Variante stellt eine so genannte Minimalvariante dar. Dabei wird auf die Inspektionsergebnisse der Kanalinspektionsfirmen vertraut und die $\mathrm{Zu}$ standserfassung als korrekt angesehen. Die TV-Inspektionsergebnisse werden ungeprüft zur Klassifizierung weiter verwendet.

Variante A1: Bei dieser Variante werden die Kanalinspektionsergebnisse durch fachlich ausgebildetes Personal überprüft. Es wird dabei aber nicht das gesamte Videomaterial durchgesehen sondern nur die dokumentierten Zustände der TV-Inspektion überprüft und gegebenenfalls korrigiert. Darauf aufbauend erfolgt dann die Zustandsklassifizierung, die basierend auf den Inspektionsergebnissen sowie den relevanten Stammdaten der Kanalisation (z. B. Haltungslängen) in der Regel computergestützt durchgeführt wird.

Der wesentliche Vorteil dieser Variante gegenüber Variante 0 ist, da Fehler bei der Zustandserfassung nicht ausgeschlossen werden können, eine zusätzliche Kontrolle der erfassten Zustände durch einen (externen) Techniker. Gegebenenfalls können falsche oder unvollständige $\mathrm{Zu}$ standsbeschreibungen korrigiert und damit Fehler in der Datengrundlage der Sanierungsplanung reduziert werden. Der gewonnene Nutzen durch die Verbesserung der Datenqualität führt aber verglichen mit Variante 0 auch zu höheren Kosten für den Auftraggeber. Allerdings stehen diese Mehrkosten in keiner Relation zu den Kosten für den Auftraggeber, wenn nachträgliche Korrekturen einer auf ungeeigneten Daten basierenden Sanierungsplanung oder -ausführung notwendig würden.

Variante A2: Diese Variante ist ähnlich der Variante A1, wobei hier aber das gesamte Videomaterial kontrolliert und gegebenenfalls korrigiert wird. Dadurch soll nicht nur wie bei Variante A1 sichergestellt werden, dass alle erfassten Zustände richtig und vollständig beschrieben sind, sondern zusätzlich auch noch gewährleistet werden, dass bei der Zustandserfassung keine Zustände übersehen wurden. Verglichen zu Variante Al wird hier die Datenqualität durch die Erhöhung der Erkennungsrate von Zuständen in der 


\begin{tabular}{lcccc} 
TABELLE 4 & & & & \\
Teilbereiche der unterschiedlichen Varianten & & Var. A2 & Var. B \\
\hline & Var. 0 & Var. A1 & + & + \\
\hline Vergabe & + & + & + & + \\
\hline Kanalreinigung & + & + & + & + \\
\hline Kanal-TV-Inspektion & + & + & - & + \\
\hline QM-Aufpreis & - & - & - & - \\
\hline $\begin{array}{l}\text { Videokontrolle teilweise } \\
\text { (nur Zustände) }\end{array}$ & - & + & + & - \\
\hline Videokontrolle gesamt & - & - & + & + \\
\hline Zustandsklassifikation & + & + & &
\end{tabular}

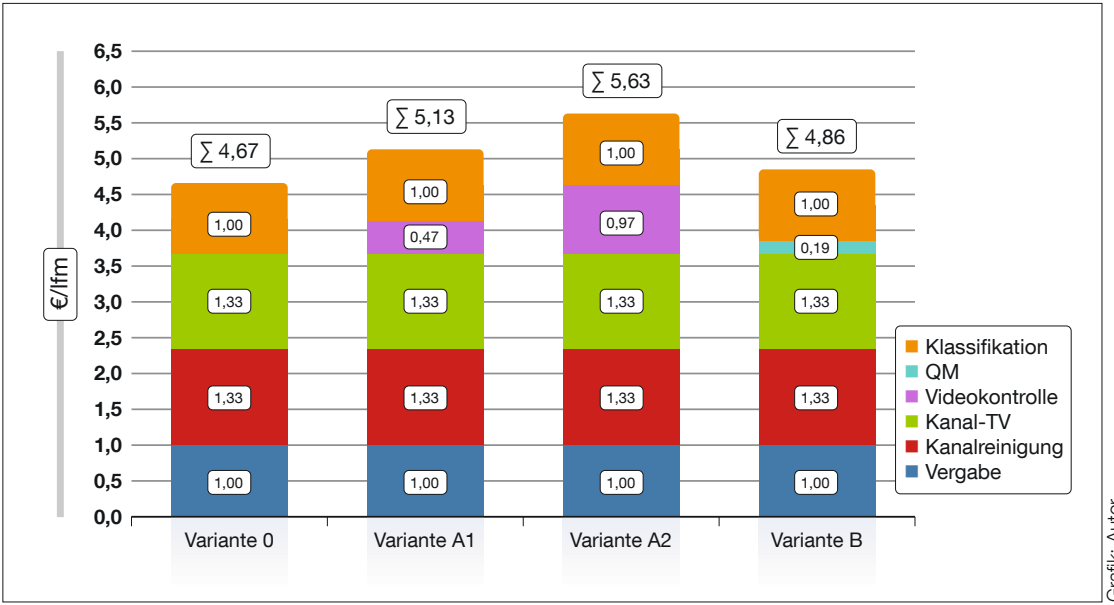

Abb. 1: Kostenvergleich der einzelnen Varianten bei Basisbedingungen. (Plihal 2009)

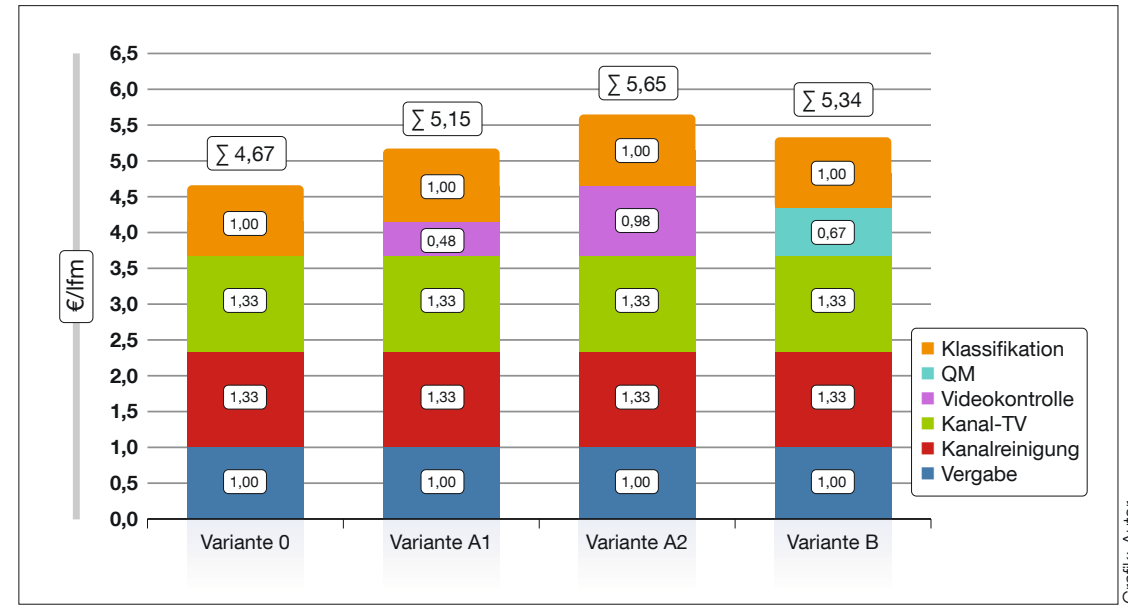

Abb. 2: Kostenvergleich der einzelnen Varianten bei geänderter durchschnittlicher Projektdauer. (Plihal 2009)

Haltung noch verbessert bzw. der Nutzen der TV-Inspektion weiter vergrößert. Dies ist allerdings wieder mit steigenden Kosten für den Auftraggeber verbunden.

Nachdem ein (externer) Techniker das gesamte Videomaterial geprüft hat, erfolgt darauf basierend dann die Zustandsklassifizierung.

Variante B: Diese Variante kann derzeit noch nicht in der Praxis durchgeführt wer- men und führt unangekündigte Baustellenbesuche durch. Eine vergleichbare Einrichtung wäre in Deutschland z. B. die Gütesicherung Kanalbau mit dem RALGütezeichen 961 mit der Beurteilungsgruppe I für Inspektion (Güteschutz Kanalbau e.V., 2009). Ziel des QM ist es, durch die laufenden Überprüfungen die Qualität der Kanalinspektionen auf ein gewünschtes Niveau zu heben und dadurch auf eine aufwändige Nachkontrolle der Ergebnisse der TV-Befahrungen verzichten zu können. Die abschließende Zustandsklassifizierung erfolgt auch bei dieser Variante durch fachlich ausgebildetes Personal wie z. B. externe Techniker.

Die einzelnen Teilbereiche der derzeit üblichen Varianten 0, A1 und A2 sowie die zukünftig mögliche Variante B sind in Tabelle 4 zusammenfassend dargestellt. Die mit einem Pluszeichen markierten Arbeitsbereiche finden in der entsprechenden Variante Anwendung, jene mit einem Minuszeichen nicht.

\section{Ergebnisse}

Die einzelnen Kostenanteile von der Auftragsvergabe über die Kanalinspektion bis hin zur Klassifizierung sowie die Gesamtkosten pro Laufmeter Kanal für die einzelnen Varianten sind in den nachfolgenden Diagrammen dargestellt. Als Ausgangsbasis für diese Diagramme wurden die unter 2.3.1 beschriebenen Annahmen herangezogen.

\subsection{Kostenvergleich mit Basisbedingungen}

Die erwähnten Annahmen stellen bei den durchgeführten Untersuchungen die Basisbedingungen dar. Aus Abbildung 1 ist ersichtlich, dass die Variante 0 (Minimalvariante) die günstigste Variante ist, gefolgt von Variante B, Variante Al und Variante A2. Die Gesamtkosten von der Vergabe bis zur Klassifizierung betragen zwischen $4,67 €$ pro Laufmeter Kanal (Variante 0 ) und 5,63€ pro Laufmeter Kanal (Variante A2).

\subsection{Kostenvergleich bei Veränderung der durchschnittlichen Projektdauer} künftig mögliche Alternative zu den oben angeführten Varianten dar. Dabei wird angenommen, dass die Kanalinspektion nur durch entsprechend qualifizierte Fachfirmen durchgeführt wird, die freiwillig ein System zur Qualitätssicherung (QS) anwenden. Ein QM-Institut macht hierbei laufend begleitende Kontrollen und Nachschulungen bei den Kanalinspektionsfir-
Die Annahmen, die zu Beginn getroffen wurden, gingen von einer durchschnittlichen Projektdauer von 20 Tagen aus. Wird die Projektdauer von 20 auf 5 Tage reduziert aber weiterhin pro Projekt mindestens 1 Tag begutachtet, so erkennt man, wie in Abbildung 2 dargestellt, dass Vari- 


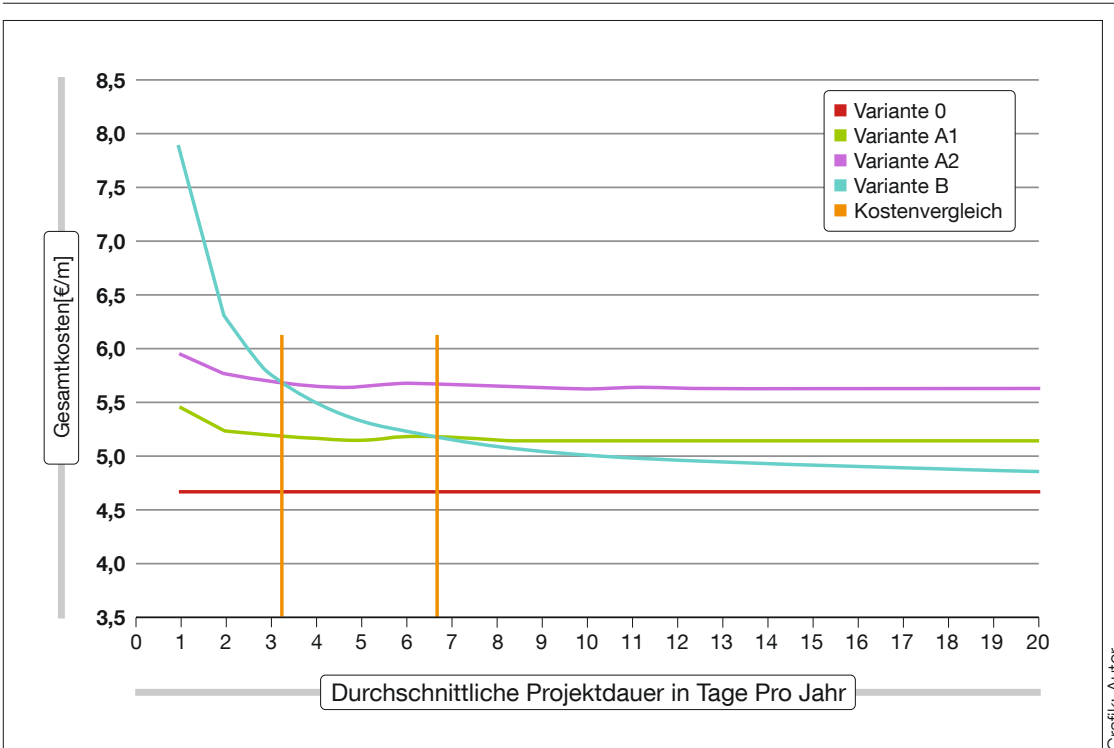

Abb. 3: Kostenentwicklung bei den einzelnen Varianten mit steigender Projektdauer. (Plihal 2009)

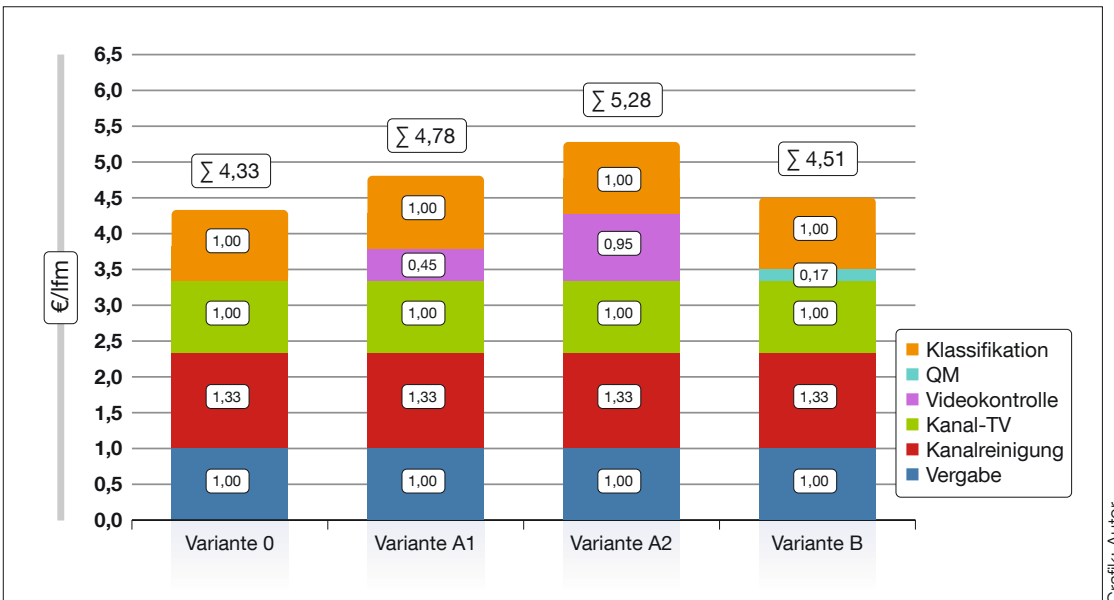

Abb. 4: Kostenentwicklung bei den einzelnen Varianten mit geänderter Laufmeterleistung der KI. (Plihal 2009)

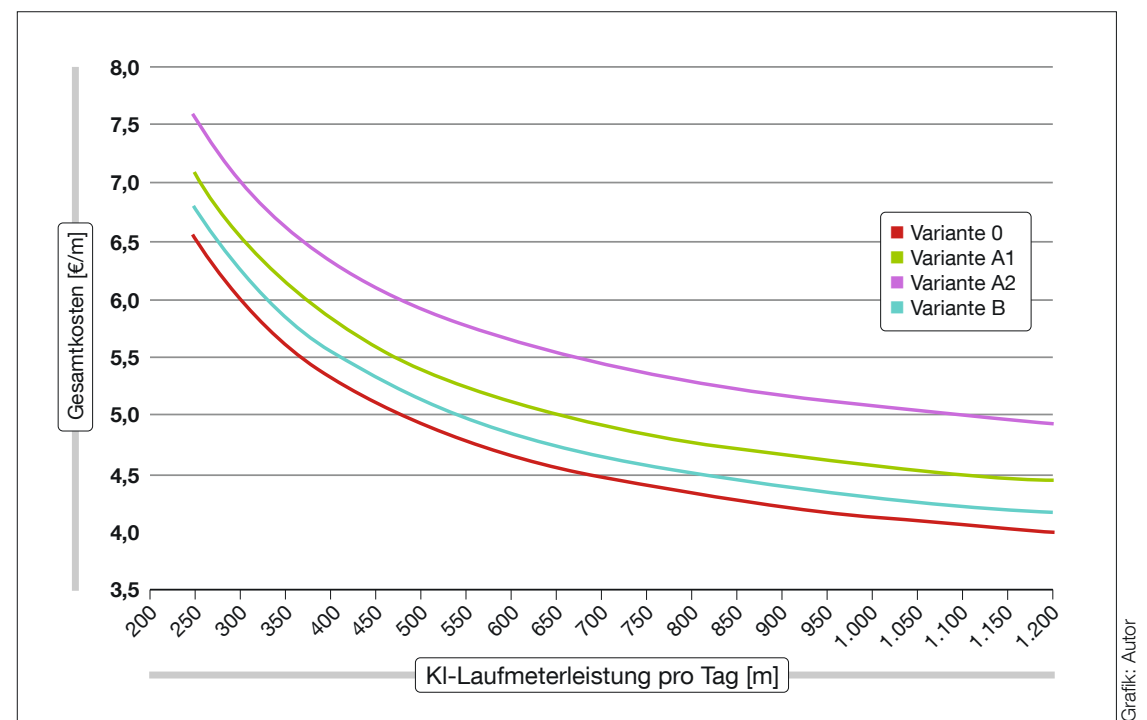

Abb. 5: Kostenentwicklung bei den einzelnen Varianten mit steigender Laufmeterleistung der KI. (Plihal 2009) ante Al günstiger als Variante $\mathrm{B}$ ist. Bei den beiden anderen Varianten 0 und A2 hat sich in der Rangfolge nichts geändert. Die Kosten pro Laufmeter haben sich erhöht, ausgenommen bei Variante 0 .

Da die Projektdauer für einzelne Kanalinspektionsfirmen während eines Kalenderjahres unterschiedlich ist und je nach Projekt variiert, ändern sich auch die Kostenanteile der einzelnen Varianten. Variiert man die durchschnittliche Projektdauer der Kanalinspektion, so erkennt man in Abbildung 3, dass die anfänglich hohen Kosten von Variante B sehr schnell sinken und ca. bei Tag 3 äquivalent mit Variante A2 und bei Tag 7 äquivalent mit Variante Al sind. Bei mehr als 7 Tagen durchschnittlicher Projektdauer ist Variante B neben Variante 0 die kostengünstigste Variante.

\subsection{Kostenvergleich bei Veränderung} der durchschnittlichen täglichen Laufmeterleistung der Kanalinspektion

Die Laufmeterleistung kann von Projekt zu Projekt sowie auch im selben Projekt variieren. In Abbildung 4 wurde die Laufmeterleistung der Kanalinspektion von ursprünglich $600 \mathrm{~m}$ auf $800 \mathrm{~m}$ erhöht. Man erkennt, dass sich die Rangfolge der einzelnen Varianten im Vergleich zu den Basisbedingungen nicht geändert hat, jedoch sind die Kosten pro Laufmeter Kanal bei allen Varianten insgesamt gesunken.

In Abbildung 5 ist ersichtlich, dass sich auch bei Variation einer täglichen Laufmeterleistung beginnend bei $250 \mathrm{~m}$ bis $1.200 \mathrm{~m}$ an der Rangfolge der einzelnen Varianten weiterhin nichts ändert.

\subsection{Kostenvergleich bei Veränderung der Anzahl der Zustände pro Haltung}

Die letzte Variable, die bei Kanalinspektionen starken Schwankungen unterworfen ist, ist die Anzahl der aufzunehmenden Zustände pro Haltung. In Abbildung 6 wurde die Anzahl der aufzunehmenden Zustände von ursprünglich 10 auf 20 erhöht. Auch hier erkennt man, dass sich die Rangfolge der einzelnen Varianten nicht geändert hat. Die Kosten pro Laufmeter Kanal haben sich mit Ausnahme von Variante 0 bei allen Varianten leicht erhöht.

Werden die Zustände pro Haltung beginnend bei einem Zustand bis insgesamt 20 Zustände variiert, so erkennt man in Abbildung 7, dass bei einem Zustand pro Haltung Variante A1 günstiger ist als Vari- 


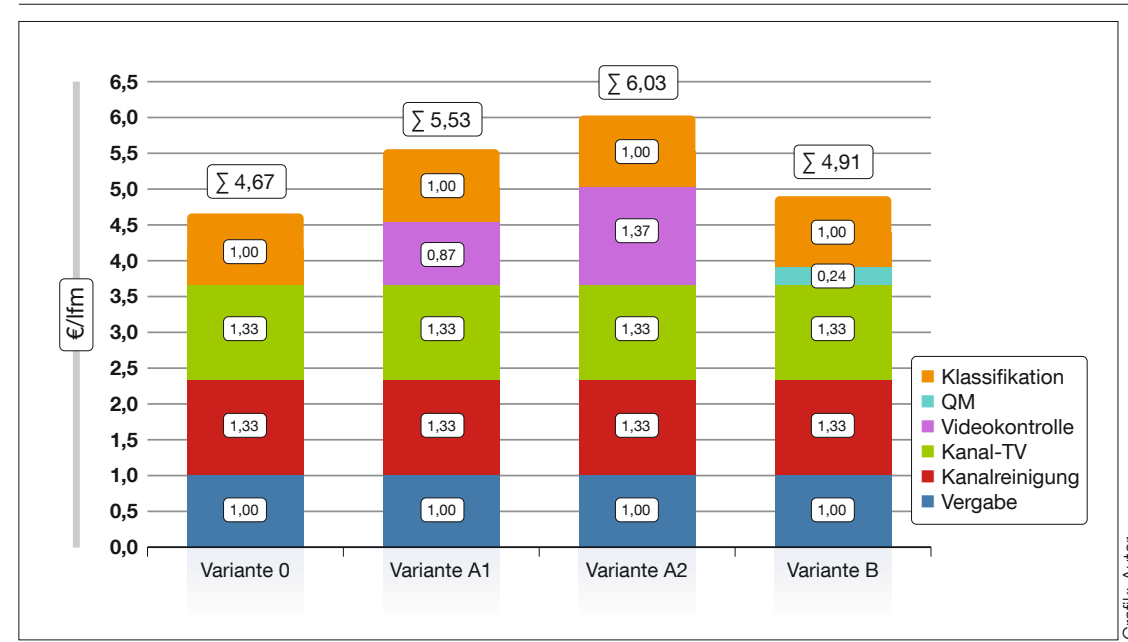

Abb. 6: Kostenentwicklung bei den einzelnen Varianten mit geänderter Anzahl an Zuständen je Haltung. (Plihal 2009)

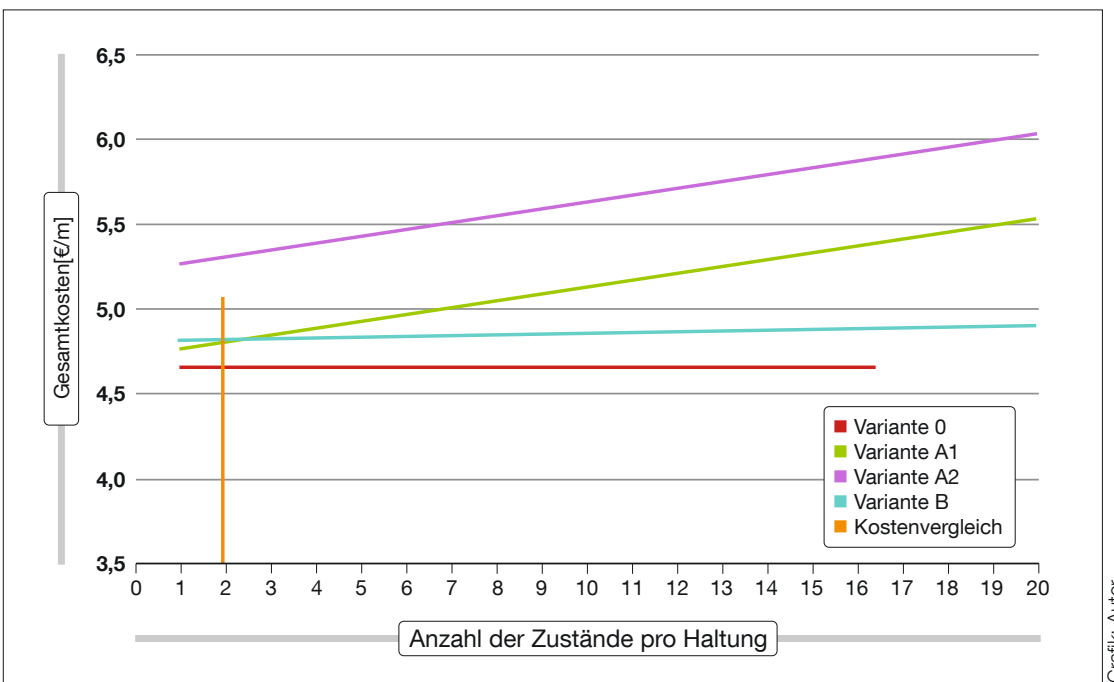

Abb. 7: Kostenentwicklung bei den einzelnen Varianten mit steigender Anzahl an Zuständen je Haltung und Kostengleichheit bei Varianten A1 und B. (Plihal 2009)

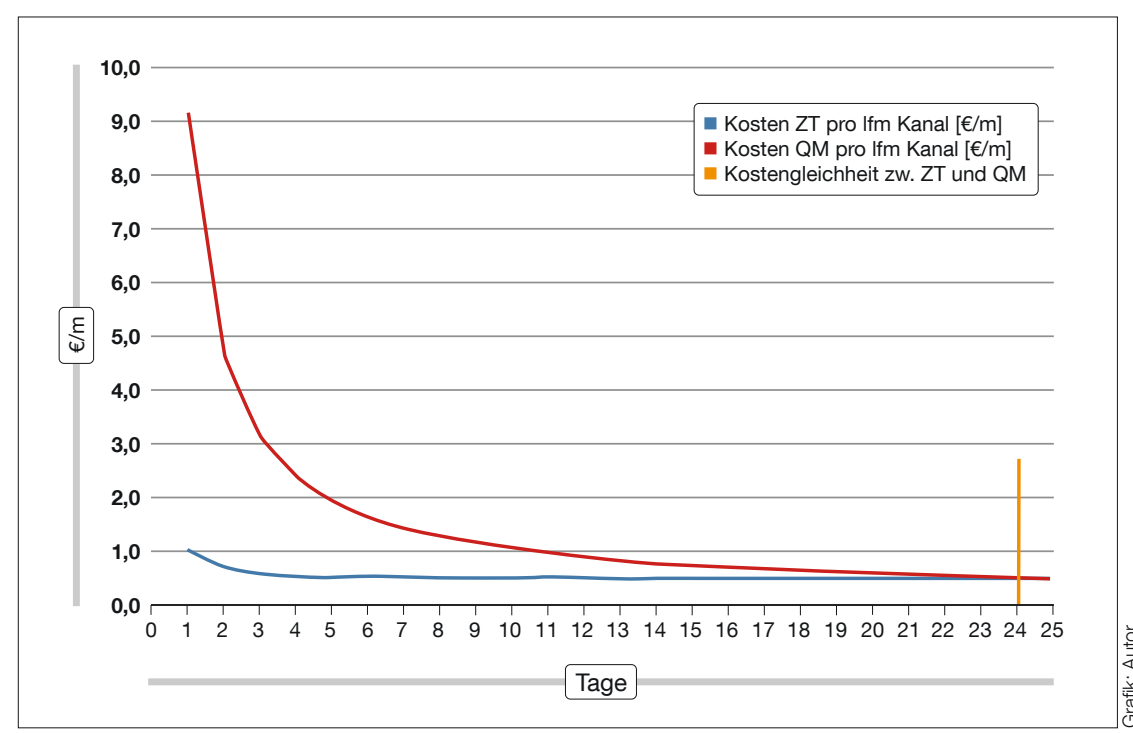

Abb. 8: Vergleich der Varianten A1 und Variante B auf 1 Projekt bezogen. (Plihal 2009) ante B. Bei 2 Zuständen pro Haltung besteht Kostengleichheit zwischen den beiden Varianten. Des Weiteren erkennt man, dass die Kosten pro Laufmeter Kanal bei den Varianten A1 und A2 wesentlich stärker ansteigen als bei Variante B. Bei Variante 0 ergibt sich keine Änderung in den Kosten bei Erhöhung der Anzahl der Zustände pro Haltung.

\subsection{Projektbezogener \\ Variantenvergleich}

Bei den oben beschriebenen Untersuchungen wurden bei Variante B die Kosten für die jährlichen Baustellenbesuche und Schulungen anteilig auf die angenommenen 10 Projekte bzw. Aufträge pro Jahr aufgeteilt. In Abbildung 8 sind sämtliche Kosten eines begleitenden QM nun auf ein einziges Projekt bezogen und die sich daraus ergebenden Laufmeterkosten in $\mathrm{Ab}$ hängigkeit zur Projektdauer dargestellt (Kurve Kosten QM). Zum Vergleich ist zusätzlich auch die Kostenkurve der Videokontrolle durch einen Techniker gemäß Variante Al abgebildet (Kurve Kosten ZT).

Aus der Abbildung ist ersichtlich, dass unter den getroffenen Annahmen ab einer Mindestprojektdauer von 25 Tagen auch bei einem Einzelprojekt die Durchführung eines begleitenden QM kosteneffizienter als Variante Al wäre.

Auf die Darstellung von Variante A2 wurde verzichtet, da bei ihr durch die Begutachtung der gesamten Videos die Kosten pro Laufmeter höher liegen als bei Variante A1. Im Vergleich mit Variante B würde das zu einer Verkürzung der Mindestprojektdauer führen.

\section{Schlussfolgerungen}

Die Variante 0 erwies sich klarerweise bei allen Untersuchungen als die mit den geringsten Kosten, weil keine Überprüfung der Ergebnisse der TV-Inspektion vor der Zustandbewertung stattfindet. Die Erfahrungen bei der TV-Inspektion zeigen allerdings, dass Fehler bei der Zustandserfassung passieren. Preise, die oftmals weit unter den zu erwartenden Kosten für eine qualitative hochwertige TV-Inspektion liegen, steigern die Häufigkeit von fehlerhaften und unvollständigen Zustandserfassungen.

Das unmittelbare Verwenden der Inspektionsprotokolle für die eigentliche $\mathrm{Zu}$ standsklassifizierung ohne jegliche Überprüfung oder ein begleitendes QM kann hier fatale Folgen auf die Sanierungspla- 
nung haben. Die Folgekosten für eine falsch gewählte oder fehlerhafte Sanierung können die Einsparungen im Zuge der Zustandserfassung und -bewertung dann jedenfalls bei weitem übersteigen. Aus diesen Gründen sollte Variante 0 als Vorgehensweise für eine sinnvolle und effiziente Grundlagenschaffung der Sanierungsplanung nicht in Betracht gezogen werden.

Verglichen mit Variante 0 sind die Varianten A1 bzw. A2 aufgrund der zusätzlichen Begutachtung der erfassten Zustände bzw. der gesamten Inspektionsvideos durch einen Techniker auf höherem qualitativen Niveau und daher als Grundlage für die Sanierungsplanung besser geeignet.

Bei Variante A2 ist verglichen mit Variante Al die Erkennungsrate aller Zustände einer Haltung durch die zusätzliche Kontrolle des gesamten Videomaterials durch einen Techniker höher. Allerdings erhöhen sich dadurch auch die Kosten.

Variante B hat gegenüber den Varianten A1 und A2 bei den gewählten Annahmen und bei Einzelbetrachtung für Projektdauern über sieben Tage finanzielle Vorteile. Auch wenn bei Variante B die Inspektionsergebnisse nur mehr stichprobenweise kontrolliert werden, können durch die begleitenden Überprüfungen von Beginn der TV-Inspektion an sowie die regelmäßigen Schulungen des relevanten Personals die erforderlichen Qualitätsstandards bei der TV-Inspektion gewährleistet werden.

Beim projektbezogenen Variantenvergleich erkennt man, dass sich durch das
QM hohe Anfangs- bzw. Fixkosten ergeben, die sich im Vergleich zu Variante Al erst bei 25 Tagen Projektdauer rechnen. Diese hohen Anfangskosten sind einerseits durch die Kosten des QM für unangekündigte Baustellenbesuche sowie andererseits durch die Kosten des QM für zusätzliche Schulungen bedingt.

Allgemein könnte durch Adaptierung dieser Faktoren die QM-Kosten deutlich reduziert und eine frühere Kostengleichheit im Vergleich zu den Varianten Al und A2 erreicht werden. Auch die Gleichsetzung des Stundensatzes von TechnikerIn und QM-Person sowie des Faktors für die Begutachtung der Kanalinspektionsvideos führt zu einer früheren Kostenäquivalenz zwischen den Varianten.

Durch Analyse einer ständigen QMBegleitung der Kanalinspektionsfirmen, dargestellt als Variante B, konnte unter den getroffenen Annahmen gezeigt werden, dass die Kosten ab einer durchschnittlichen Projektdauer von mindestens 7 Tagen geringer sind als die Varianten Al, ab 4 Tagen ist Variante B bereits günstiger als Variante A2.

Die Untersuchungen zeigen, dass ein begleitendes QM bei der TV-Inspektion Vorteile in finanzieller Hinsicht bei gleichzeitiger Gewährleistung der notwendigen hohen Qualitätsstandards bei der Zustandserfassung bringen kann.

\section{Zusammenfassung}

Ausgehend von nationalen und internationalen Erfahrungen zu Erkennungsraten

\section{LITERATUR}

BMLFUW (2006) Kommunale Siedlungswasserwirtschaft - Förderungsrichtlinien 1999 in der Fassung 2006. Wien

BÖLKE KP, ERTL T (2007) Kritische Betrachtung der Zustandsbewertung von TV-Inspektionen nach EN 13508-2. Wiener Mitteilungen, Band 203, C1-C19, Wien

GANGL G, ERTL T, KRETSCHMER F, KAINZ H, HABERL R (2006) Development of a Guideline for Sewer Operation and Maintenance in Austria. In: Conference Proceedings 2 nd International IWA Conference on Sewer Operation and Maintenance, p. 215-222, October 26-28, 2006, Vienna

GÜTESCHUTZ KANALBAU e.V. (2009) Herstellung und Instandhaltung von Abwasserleitungen und -kanälen. Gütesicherung Kanalbau RAL-GZ 961. Online im Internet: URL: http:// RAL-GZ 961. Online im Internet: URL: http://
www.kanalbau.sslh.net/cnt/Oeffentlichkeitsarbeit/pdf/RAL-GZ_961_2009.pdf [Abruf: 27.08.2009]

IKT (2009) Bayern: 3,6 Mrd. EUR Kanalsanierungsbedarf. eNewsletter Juli 2009. Online im Internet: URL: http://www.ikt.de/index. php?doc=1052 [Abruf: 27.08.09] php?
JUNG P (2005) Anforderungen an die Inspektion als Basis einer sinnvollen Sanierungsplanung 18. Lindauer Seminar. Tagungsband auf CDRom.

Lebensministerium (Hrsg.) (2006) KANFUNKÜberprüfung, Bewertung und Sicherstellung de Funktionsfähigkeit von Kanalisationsanlagen in Österreich, Endbericht. Wien

KOCH A (2009) Umstieg auf das Kodiersystem der DIN EN 13508-2 - Anforderungen und Aufgaben, 11. Dresdner Abwassertagung

PLIHAL H (2009) Evaluierung von Maßnahmen zur Qualitätssicherung bei der kamerabasierten Kanalinspektion. Diplomarbeit am Institut für Siedlungswasserbau, Industriewasserwirtschaf und Gewässerschutz, Universität für Bodenkultur Wien

VDRK (2009) Ausweis für Qualität und Seriosität. VDRK stellt „Qualitätspass“ für Inspektion und Dichtheitsprüfung in der Grundstücksentwässerung vor. Pressemitteilung. Online im Internet: URL: http://www.vdrk.de/index. php?action=verband/struktur/fachbeirat [Abruf: 27.08.09]

WINKLER U (2003) Welche Informationen benötigt die Kanal-Inspektion zukünftig?, 16. Lindauer Seminar. Tagungsband auf CD-ROM und Fehlerpotenzialen bei TV-Inspektionen wurde eine Analyse der Arbeitsschritte im Zuge der Grundlagenermittlung für die Sanierungsplanung von Kanalisationen durchgeführt. Unter definierten Randbedingungen wurde eine Kosten-Nutzen-Analyse von Vorgehensweisen bei unterschiedlichen qualitätssichernden Maßnahmen (Varianten 0, Al, A2 und B) erstellt. Die Randbedingungen wurden weiters einer Sensitivitätsanalyse unterzogen.

Die Ergebnisse zeigen, dass die Variante B mit dem begleitenden QM (Qualitätsmanagement) gegenüber den Varianten A1 (Überprüfung der bei der TV-Inspektion erfassten Zustände) und A2 (Überprüfung des gesamten Videomaterials der TV-Inspektion) bei den gewählten Annahmen und bei Einzelbetrachtung für Projektdauern über vier bzw. sieben Tage finanzielle Vorteile mit sich bringt. Dabei können durch die begleitenden Überprüfungen sowie die regelmäßigen Schulungen des relevanten Personals die erforderlichen Qualitätsstandards bei der TV-Inspektion gewährleistet werden.

Insbesondere bei Altbestand und zu erwartendem Sanierungsbedarf werden dadurch Bedingungen geschaffen, unter denen eine qualitativ hochwertige TV-Inspektion die erforderliche Basis für eine Sanierungsplanung erbringen kann.

Die berechneten Kosten sind theoretische Preise. Ausschreibungen der hier berechneten Varianten können aus verschiedenen Gründen davon abweichende Preise erzielen. Der Unterschied zu den Marktpreisen (v. a. bei Billigstbieterverfahren) ist in Relation zu den zu erwartenden Sanierungskosten aber vernachlässigbar, bei gleichzeitiger Steigerung der Qualität der erbrachten Leistungen.

Die Pflichten des/der Auftraggebers/ Auftraggeberin, wie z. B. die Überprüfung der Erbringung der vertraglichen Leistungen, können durch die vorgeschlagenen begleitenden qualitätssichernden Maßnahmen sehr kosteneffizient erfüllt werden.

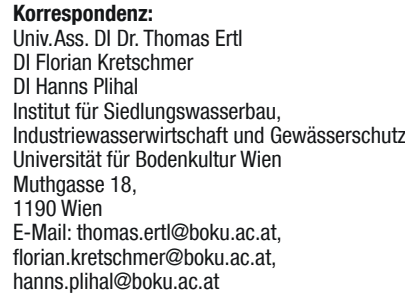

